\title{
Notes on eigenvalues of Laplacian acting on $p$-forms
}

\author{
By Satoshi AsadA
}

(Received December 5, 1978)

\section{Introduction.}

By generalizing the method of Payne-Pólya-Weinberger ([4]), for a compact domain on a minimal hypersurface in the Euclidean space, S. Y. Cheng [2]] proved an inequality between successive eigenvalues of the Laplacian acting on $C^{\infty}$-functions on this domain. On the other hand, M. Maeda ([3]) has got a similar result for a compact minimal submanifold with or without boundary in the unit sphere. The purpose of the present note is to present a similar inequality between successive eigenvalues of the Laplacian acting on $p$-forms (resp. 1-forms) on a compact and oriented minimal hypersurface (resp. minimal submanifold of any codimension) without boundary in the unit sphere.

Let $M$ be an $m(\geqslant 2)$-dimensional compact and oriented Riemannian manifold without boundary with the Riemannian metric $g$. For each $p=0$, $1, \cdots, m, A^{p}(M)$ denotes the space of all differential $p$-forms on $M$. For $\omega$, $\eta \in A^{p}(M)$, we can define a $C^{\infty}$-function $(\omega \mid \eta)$ on $M$ as follows : $(\omega \mid \eta)$ is locally given by

$$
(\omega \mid \eta)=\sum_{\substack{j_{1} \cdots j_{p} \\ k_{1} \cdots k_{p}=1}}^{m} \omega_{j_{1} \cdots j_{p}} \eta_{k_{1} \cdots k_{p}} g^{j_{1} k_{1} \cdots g^{j_{p} k_{p}}}
$$

where we put $g_{j k}=g\left(\frac{\partial}{\partial z^{j}}, \frac{\partial}{\partial z^{k}}\right)$ for a local coordinate system $\left\{z^{j} ; j=1, \cdots\right.$, $m\}$ of $M$ and $\left(g^{j k}\right)$ is the inverse matrix of $\left(g_{j k}\right)$. The inner product $\langle$, on $A^{p}(M)$ is defined by $\langle\omega, \eta\rangle:=\int_{M}(\omega \mid \eta) d V_{M}$. Here $d V_{M}$ denotes the volume form of $M$. We put $\|\omega\|:=\sqrt{\langle\omega, \omega\rangle}$. Let $d: A^{p}(M) \rightarrow A^{p+1}(M)$ be the operator of exterior differentiation and $\delta: A^{p+1}(M) \rightarrow A^{p}(M)$ be the adjoint operator of $d$ with respect to $\langle$,$\rangle . Then the Laplace-Beltrami operator$ (Laplacian for short) $\Delta$ acting on $p$-forms is defined by $\Delta:=d \delta+\delta d: A^{p}(M)$ $\rightarrow A^{p}(M)$. The Laplacian is elliptic and a self-adjoint operator. Thus all eigenvalues of the Laplacian form a discrete infinite sequence:

$$
0=\lambda_{0}^{p} \leqslant \lambda_{1}^{p} \leqslant \cdots \leqslant \lambda_{n}^{p} \leqslant \cdots ; \lambda_{n}^{p} \longrightarrow \infty
$$

where each $\lambda_{n}^{p}$ is repeated as many time as its multiplicity. Let $\left\{\varphi_{r} ; r=\right.$ 
$1,2, \cdots\}$ be a complete orthonormal base in $A^{p}(M)$ consisting of eigenforms of the Laplacian. Then we have the so-called minimum principle.

Minimum principle.

$$
\begin{aligned}
& \lambda_{n+1}^{p}=\inf \left\{\langle\Delta \omega, \omega\rangle /\|\omega\|^{2} ; \omega \in A^{p}(M), \omega \neq 0,\left\langle\omega, \varphi_{r}\right\rangle=0\right. \\
& \quad \text { for all } r=1, \cdots, n\} .
\end{aligned}
$$

In Section 2, we shall prove the following theorem.

THEOREM 1. Let $M$ be an $m(\geqslant 2)$-dimensional compact and oriented Riemannian manifold without boundary minimally immersed in the unit $(m+1)$-sphere. Then for each numbers $n \geqslant 1$ and $1 \leqslant p \leqslant m$, we have the inequality:

$$
\lambda_{n+1}^{p}<\lambda_{n}^{p}+\frac{2(\Omega+1)}{n m}\left\{\sum_{r=1}^{n} \lambda_{r}^{p}+p n\left(p-m+S(M)^{2}\right)\right\}+\left(\frac{1}{\Omega}+1\right) m .
$$

Here $S(M)$ denotes the maximum of the length of the second fundamental form of the immersion and $\Omega$ is a free parameter such that $\Omega \geqslant 1$.

In Section 3, we shall prove the following theorem.

THEOREM 2. Let $M$ be an $m(\geqslant 2)$-dimensional compact and oriented Riemannian manifold without boundary minimally immersed in the unit sphere (of any codimension). Then for each number $n \geqslant 1$, we have the inequality:

$$
\lambda_{n+1}^{1}<\lambda_{n}^{1}+\frac{2(\Omega+1)}{n m}\left\{\sum_{t=1}^{n} \lambda_{t}^{1}-n r(M)\right\}+\left(\frac{1}{\Omega}+1\right) m .
$$

Here $r(M)$ denotes the minimum of the Ricci curvature of $M$ and $\Omega$ is a free parameter such that $\Omega \geqslant 1$.

\section{Estimate of $\lambda_{n+1}^{p}$.}

Let $M$ be an $m(\geqslant 2)$-dimensional compact and oriented Riemannian manifold without boundary minimally immersed in the unit $(m+1)$-sphere $S^{m+1} \subset E^{m+2}$. Let $\left\{x^{\alpha} ; \alpha=1, \cdots, m+2\right\}$ denote an orthonormal coordinate system in $E^{m+2}$. We also denote by $x^{\alpha}$ the restriction of $x^{\alpha}$ to $M$. The next is well known (cf. T. Takahashi [6]).

Lemma 2.1. $\Delta x^{\alpha}=m x^{\alpha}(1 \leqslant \alpha \leqslant m+2)$.

Fix arbitrary numbers $1 \leqslant p \leqslant m$ and $n \geqslant 1$. We use the following convention on the range of indices unless otherwise stated: $i, j, k, l, j_{1}, \cdots$, $j_{p}=1, \cdots, m ; \mu, \nu=1, \cdots, p ; r, t=1, \cdots, n$ and $\alpha=1, \cdots, m+2$.

Lemma 2.2. For any $\omega \in A^{p}(M)$, we have the inequality 


$$
\langle\nabla \omega, \nabla \omega\rangle \leqslant\langle\Delta \omega, \omega\rangle+p\left(p-m+S(M)^{2}\right) \cdot\|\omega\|^{2} .
$$

Here $\nabla$ denotes the Levi-Civita connection defined by the Riemannian metric $g$ of $M$.

Proof. It is known that $\Delta \omega$ is locally expressed in terms of $\nabla$, the Riemannian curvature tensor $R$ of $M$ and the Ricci tensor $\rho$ of $M$ (cf. G. de Rham [5], p. 131). That is, if $p \geqslant 2$, then we have

$$
\begin{aligned}
(\Delta \omega)_{j_{1} \cdots j_{p}}= & -\sum_{i} \nabla^{i} \nabla_{i} \omega_{j_{1} \cdots j_{p}}-\sum_{k, \nu}(-1)^{\nu} \rho_{j_{\nu}}{ }^{k} \cdot \omega_{k j_{1} \cdots \hat{j}_{\nu} \cdots j_{p}} \\
& +2 \sum_{i, k} \sum_{\mu<\nu}(-1)^{\mu+\nu} R_{j_{\nu} \cdot j_{\mu}}^{k} \cdot \omega_{i k j_{1} \cdots \hat{j}_{\mu} \cdots j_{\nu} \cdots j_{p}}
\end{aligned}
$$

and if $p=1$, then we have

$$
(\Delta \omega)_{j}=-\sum_{i} \nabla^{i} \nabla_{i} \omega_{j}+\sum_{k} \rho_{j}{ }^{k} \cdot \omega_{k} .
$$

Here $\wedge$ over $j_{\nu}$ indicates that it is omitted. Fix an arbitrary point $z_{0} \in M$. Since the codimension of $M$ in $S^{m+1}$ is equal to one, we can choose a normal coordinate system $\left\{z^{j}\right\}$ of $M$ about $z_{0}$ such that the second fundamental form $\left(S_{j k}\right)$ at $z_{0}$ is given by a diagonal matrix, i. e., $S_{j k}=\delta_{j k} \xi_{k}$. Here $\xi_{k}$ denote the principal curvatures at $z_{0}$. Then, at $z_{0}$, the equation of Gauss implies

$$
R_{j \cdot l}^{k i}=\delta_{j l} \cdot \delta_{i k}-\delta_{k l} \cdot \delta_{i j}+\delta_{j l} \cdot \delta_{i k} \cdot \xi_{l} \cdot \xi_{k}-\delta_{k l} \cdot \delta_{i j} \cdot \xi_{l} \cdot \xi_{j} .
$$

By means of $\sum_{i} \xi_{i}=0$, we have, at $z_{0}$,

$$
\rho_{j}{ }^{k}=-\sum_{i} R_{i \cdot j}^{k i}=\left(m-1-\xi_{j} \cdot \xi_{k}\right) \cdot \delta_{j k} .
$$

First we assume that $p \geqslant 2$. From (4), (6) and (7), we have

$$
-\sum_{i} \nabla^{i} V_{i} \omega_{j_{1} \cdots j_{p}}=(\Delta \omega)_{j_{1} \cdots j_{p}}+p(p-m) \cdot \omega_{j_{1} \cdots j_{p}}+\left(\sum_{\nu} \xi_{j_{\nu}}\right)^{2} \cdot \omega_{j_{1} \cdots j_{p}} .
$$

Since $\omega^{j_{1} \cdots j_{p}}=\omega_{j_{1} \cdots j_{p}}$ at $z_{0}$, we get

$$
\left(-\sum_{i} \nabla^{i} \nabla_{i} \omega \mid \omega\right)=(\Delta \omega \mid \omega)+p(p-m) \cdot(\omega \mid \omega)+\sum_{j_{1} \cdots j_{p}}\left(\sum_{\nu} \xi_{j_{\nu}}\right)^{2} \cdot\left(\omega_{j_{1} \cdots j_{p}}\right)^{2} .
$$

On the other hand, by Schwartz inequality, we have

$$
\begin{aligned}
\sum_{j_{1} \cdots j_{p}}\left(\sum_{\nu} \xi_{j_{\nu}}\right)^{2} \cdot\left(\omega_{j_{1} \cdots j_{p}}\right)^{2}=p ! \sum_{j_{1}<\cdots<j_{p}}\left(\sum_{\nu} \xi_{j_{\nu}}\right)^{2} \cdot\left(\omega_{j_{1} \cdots j_{p}}\right)^{2} \\
\quad \leqslant p ! \sum_{j_{1}<\cdots<j_{p}} p\left(\sum_{\nu} \xi_{j_{\nu}}^{2}\right) \cdot\left(\omega_{j_{1} \cdots j_{p}}\right)^{2} \leqslant p\left(\sum_{i} \xi_{i}^{2}\right) \cdot p ! \sum_{j_{1}<\cdots<j_{p}}\left(\omega_{j_{1} \cdots j_{p}}\right)^{2} \\
\quad \leqslant p \cdot S(M)^{2} \cdot(\omega \mid \omega),
\end{aligned}
$$

because $\sum_{i} \xi_{i}^{2}$ is equal to the value of the square of the length of the 
second fundamental form at $z_{0}$. Hence we have

$$
\left(-\sum_{i} \nabla^{i} \nabla_{i} \omega \mid \omega\right) \leqslant(\Delta \omega \mid \omega)+p\left(p-m+S(M)^{2}\right)(\omega \mid \omega) .
$$

By integrating both sides of the above inequality, we get by Stokes formula

$$
\begin{aligned}
\langle\nabla \omega, \nabla \omega\rangle & =\left\langle-\sum_{i} \nabla^{i} \nabla_{i} \omega, \omega\right\rangle \\
& \leqslant\langle\Delta \omega, \omega\rangle+p\left(p-m+S(M)^{2}\right) \cdot\|\omega\|^{2} .
\end{aligned}
$$

Next in the case $p=1$, by means of (5) and (7), we have a similar argument as above to obtain

$$
\langle\nabla \omega, \nabla \omega\rangle \leqslant\langle\Delta \omega, \omega\rangle+\left(1-m+S(M)^{2}\right) \cdot\|\omega\|^{2} . \quad \text { q. e. d. }
$$

REMARK 2.1. The Riemannian curvature tensor $R$ is defined by

$$
R(X, Y) Z=\left[\nabla_{X}, \nabla_{Y}\right] Z-\nabla_{[X, Y]} Z
$$

and we put

$$
R\left(\frac{\partial}{\partial z^{k}}, \frac{\partial}{\partial z^{l}}\right) \frac{\partial}{\partial z^{j}}=\sum_{i} R_{j k l}^{i} \frac{\partial}{\partial z^{i}} .
$$

The Ricci tensor $\rho$ is defined by $\rho(Y, Z)=\operatorname{Trace}(X \rightarrow R(X, Y) Z)$. Thus we have

$$
\rho_{j k}=\rho\left(\frac{\partial}{\partial z^{j}}, \frac{\partial}{\partial z^{k}}\right)=\sum_{i} R_{j i k}^{i} .
$$

REMARK 2.2. If $M$ is not totally geodesic, then by means of the inequality of J. Simons we have $S(M)^{2} \geqslant m$ (cf. B. Y. Chen [1], p. 94).

Let $\left\{\varphi_{h} ; h=1,2, \cdots\right\}$ be a complete orthonormal base in $A^{p}(M)$ consisting of eigenforms of the Laplacian. Put $a_{r t}^{\alpha}:=\left\langle x^{\alpha} \varphi_{r}, \varphi_{t}\right\rangle\left(=a_{t r}^{\alpha}\right)$ and $U_{r}^{\alpha}:=x^{\alpha} \varphi_{r}-\sum_{t} a_{r t}^{\alpha} \varphi_{t}$.

Lemma 2.3. $\sum_{\boldsymbol{\alpha}, r}\left\langle\nabla_{\operatorname{grad}\left(x^{\alpha}\right)} \varphi_{r}, U_{r}^{\alpha}\right\rangle=-(m / 2) \sum_{\boldsymbol{\alpha}, r, t}\left(a_{r t}^{\alpha}\right)^{2}$.

Proof. By means of $\sum_{\alpha}\left(x^{\alpha}\right)^{2}=1$, we have

$$
\sum_{\alpha}\left\langle\nabla_{\operatorname{grad}\left(x^{\alpha}\right)} \varphi_{r}, x^{\alpha} \varphi_{r}\right\rangle=\left\langle\nabla_{\alpha} x^{\alpha} \cdot \operatorname{grad}\left(x^{\alpha}\right) \varphi_{r}, \varphi_{r}\right\rangle=0 .
$$

On the other hand, we have

$$
\begin{aligned}
& \sum_{r}\left\langle\nabla_{g r a d}\left(x^{\alpha}\right) \varphi_{r}, \sum_{t} a_{r t}^{\alpha} \varphi_{t}\right\rangle=\sum_{r, t} a_{r t}^{\alpha}\left\langle\nabla_{g r a d}\left(x^{\alpha}\right) \varphi_{r}, \varphi_{t}\right\rangle \\
& \quad=\sum_{r, t} a_{r t}^{\alpha} \int_{M} \operatorname{grad}\left(x^{\alpha}\right) \cdot\left(\varphi_{r} \mid \varphi_{t}\right) d V_{M}-\sum_{r, t} a_{r t}^{\alpha}\left\langle\varphi_{r}, \nabla_{g r a d\left(x^{\alpha}\right)} \varphi_{t}\right\rangle \\
& \quad=\sum_{r, t} a_{r t}^{\alpha}\left\langle d x^{\alpha}, d\left(\varphi_{r} \mid \varphi_{t}\right)\right\rangle-\sum_{r, t} a_{r t}^{\alpha}\left\langle\nabla_{g r a d}\left(x^{\alpha}\right) \varphi_{r}, \varphi_{t}\right\rangle
\end{aligned}
$$


because of $a_{r t}^{\alpha}=a_{r t}^{\alpha}$. Thus we have

$$
\begin{gathered}
\sum_{r}\left\langle\nabla_{\text {grad }\left(x^{\alpha}\right)} \varphi_{r}, \sum_{t} a_{r t}^{\alpha} \varphi_{t}\right\rangle=(1 / 2) \sum_{r, t} a_{r t}^{\alpha}\left\langle\Delta x^{\alpha},\left(\varphi_{r} \mid \varphi_{t}\right)\right\rangle \\
=(m / 2) \sum_{r, t} a_{r t}^{\alpha}\left\langle x^{\alpha},\left(\varphi_{r} \mid \varphi_{t}\right)\right\rangle=(m / 2) \sum_{r, t}\left(a_{r t}^{\alpha}\right)^{2} .
\end{gathered}
$$

From (i) and (ii) we get the assertion of our lemma.

q. e. d.

Lemma 2.4. $\sum_{\alpha, r}\left\|\nabla_{\operatorname{grad}\left(x^{\alpha}\right)} \varphi_{r}\right\|^{2} \leqslant \sum_{r} \lambda_{r}^{p}+p n\left(p-m+S(M)^{2}\right)$.

Proof. Fix an arbitrary point $z_{0} \in M$. Let $\left\{z^{j}\right\}$ be a normal coordinate system of $M$ about $z_{0}$. Put $e_{j}=\left.\frac{\partial}{\partial z^{j}}\right|_{z_{0}}$, then we have, at $z_{0}$,

$$
\begin{aligned}
& \sum_{\alpha}\left(\nabla_{\operatorname{grad}\left(x^{\alpha}\right)} \varphi_{r} \mid \nabla_{\operatorname{grad}\left(x^{\alpha}\right)} \varphi_{r}\right) \\
& \quad=\sum_{\alpha, j, k} g\left(\operatorname{grad}\left(x^{\alpha}\right), e_{j}\right) \cdot g\left(\operatorname{grad}\left(x^{\alpha}\right), e_{k}\right) \cdot\left(\nabla_{j} \varphi_{r} \mid \nabla_{k} \varphi_{r}\right) \\
& \quad=\sum_{j, k} \sum_{\alpha}\left(e_{j} x^{\alpha}\right) \cdot\left(e_{k} x^{\alpha}\right) \cdot\left(\nabla_{j} \varphi_{r} \mid \nabla_{k} \varphi_{r}\right)=\sum_{j}\left(\nabla_{j} \varphi_{r} \mid \nabla_{j} \varphi_{r}\right)=\left(\nabla \varphi_{r} \mid \nabla \varphi_{r}\right)
\end{aligned}
$$

because of $\sum_{\alpha}\left(e_{j} x^{\alpha}\right) \cdot\left(e_{k} x^{\alpha}\right)=g_{j k}\left(z_{0}\right)=\delta_{j k}$. Thus, by lemma 2.2, we get

$$
\begin{aligned}
& \sum_{\alpha, r}\left\|\nabla_{\text {grad }\left(x^{\alpha}\right)} \varphi_{r}\right\|^{2}=\sum_{r}\left\langle\nabla \varphi_{r}, \nabla \varphi_{r}\right\rangle \\
& \quad \leqslant \sum_{r}\left\{\left\langle\Delta \varphi_{r}, \varphi_{r}\right\rangle+p\left(p-m+S(M)^{2}\right) \cdot\left\|\varphi_{r}\right\|^{2}\right\} \\
& \quad=\sum_{r} \lambda_{r}^{p}+p n\left(p-m+S(M)^{2}\right) .
\end{aligned}
$$

We see easily that $\left\langle U_{r}^{\alpha}, \varphi_{t}\right\rangle=0$ for any $\alpha, r, t$. Thus by means of the minimum principle, we get

$$
\lambda_{n+1}^{p} \sum_{\alpha, r}\left\|U_{r}^{\alpha}\right\|^{2} \leqslant \sum_{\alpha, r}\left\langle\Delta U_{r}^{\alpha}, U_{r}^{\alpha}\right\rangle .
$$

If $U_{r}^{\alpha}=0$ for any $\alpha$ and $r$, then $x^{\alpha} \varphi_{r}=\sum_{t} a_{r t}^{\alpha} \varphi_{t}$. So $1=\sum_{\alpha}\left\langle x^{\alpha} \varphi_{r}, x^{\alpha} \varphi_{r}\right\rangle=\sum_{\alpha, t}\left(a_{r t}^{\alpha}\right)^{2}$ for all $r$. Hence $n=\sum_{\alpha, r, t}\left(a_{r t}^{\alpha}\right)^{2}$. This contradicts lemma 2.3. Therefore we have $\sum_{\alpha, r}\left\|U_{r}^{\alpha}\right\|^{2}>0$. On the other hand, we have $\sum_{\alpha, r}\left\|U_{r}^{\alpha}\right\|^{2}=n-\sum_{\alpha, r, t}\left(a_{r t}^{\alpha}\right)^{2}$. Put $A:=\sum_{\alpha, r, t}\left(a_{r t}^{\alpha}\right)^{2}$, then from (8) we get

$$
\lambda_{n+1}^{p} \leqslant\left(\sum_{\boldsymbol{a}, r}\left\langle\Delta U_{r}^{\alpha}, U_{r}^{\alpha}\right\rangle\right) /(n-A) .
$$

It is known that for $f \in A^{\circ}(M)$ and $\omega \in A^{p}(M)$, we have $\Delta(f \omega)=(\Delta f) \omega+$ $f \Delta \omega-2 V_{\text {grad(f) }} \omega$ (cf. G. de Rham [5], p. 129). Therefore we get

$$
\begin{aligned}
\Delta U_{r}^{\alpha} & =\left(\Delta x^{\alpha}\right) \varphi_{r}+x^{\alpha} \Delta \varphi_{r}-2 V_{g r a d}\left(x^{\alpha}\right) \varphi_{r}-\sum_{t} a_{r t}^{\alpha} \Delta \varphi_{t} \\
& =\left(m+\lambda_{r}^{p}\right) x^{\alpha} \varphi_{r}-2 \nabla_{g r a d\left(x^{\alpha}\right)} \varphi_{r}-\sum_{t} a_{r t}^{\alpha} \lambda_{t}^{p} \varphi_{t}
\end{aligned}
$$


and

$$
\begin{aligned}
\left\langle\Delta U_{r}^{\alpha}, U_{r}^{\alpha}\right\rangle & =\left(m+\lambda_{r}^{p}\right)\left\langle x^{\alpha} \varphi_{r}, U_{r}^{\alpha}\right\rangle-2\left\langle\nabla_{g r a d\left(x^{\alpha}\right)} \varphi_{r}, U_{r}^{\alpha}\right\rangle \\
& \leqslant\left(m+\lambda_{n}^{p}\right) \cdot \|\left. U_{r}^{\alpha}\right|^{2}-2\left\langle\nabla_{\operatorname{grad}\left(x^{\alpha}\right)} \varphi_{r}, U_{r}^{\alpha}\right\rangle .
\end{aligned}
$$

Hence, by means of lemma 2.3, we have

$$
\sum_{\alpha, r}\left\langle\Delta U_{r}^{\alpha}, U_{r}^{\alpha}\right\rangle \leqslant\left(m+\lambda_{n}^{p}\right)(n-A)+m A
$$

From (9), we get

$$
\begin{aligned}
& \lambda_{n+1}^{p}-\lambda_{n}^{p}-m \leqslant m A /(n-A)=-m+n m /(n-A) . \\
& \lambda_{n+1}^{p}-\lambda_{n}^{p} \leqslant n m /(n-A) .
\end{aligned}
$$

By means of Schwartz inequality, lemma 2.3 and lemma 2.4, we have

$$
\begin{aligned}
m^{2} A^{2} / 4 & =\left(\sum_{\alpha, r}\left\langle\nabla_{\operatorname{grad}\left(x^{\alpha}\right)} \varphi_{r}, U_{r}^{\alpha}\right\rangle\right)^{2} \\
& \leqslant\left(\sum_{\boldsymbol{\alpha}, r}\left\|\nabla_{\operatorname{grad}\left(x^{\alpha}\right)} \varphi_{r}\right\|^{2}\right) \cdot\left(\sum_{\boldsymbol{\alpha}, r}\left\|U_{r}^{\alpha}\right\|^{2}\right) \\
& \leqslant\left\{\sum_{r} \lambda_{r}^{p}+p n\left(p-m+S(M)^{2}\right)\right\}(n-A) .
\end{aligned}
$$

If $A=0$, then by (10) we have $\lambda_{n+1}^{p}-\lambda_{n}^{p}-m \leqslant 0$.

This estimate is sharper than the case $A \neq 0$ because of $A \geqslant 0$. So hereafter we consider the case $A \neq 0$. Form (10) and [12), we get

$$
\lambda_{n+1}^{p}-\lambda_{n}^{p}-m \leqslant 4\left\{\sum_{r} \lambda_{r}^{p}+p n\left(p-m+S(M)^{2}\right\} / m A .\right.
$$

Put $X:=\lambda_{n+1}^{p}-\lambda_{n}^{p}-m$ and $K:=\sum_{r} \lambda_{r}^{p}+p n\left(p-m+S(M)^{2}\right)$, then (11) and (13) mean

$$
\begin{aligned}
& X+m \leqslant n m /(n-A) . \\
& X \leqslant 4 K / m A .
\end{aligned}
$$

From $\left(11^{\prime}\right)$ and $\left(13^{\prime}\right)$, we have

$$
n m X^{2}-4 K X-4 K m \leqslant 0 .
$$

Thus we get

$$
\begin{aligned}
X & \leqslant 2\left(K+\sqrt{K^{2}+K n m^{2}}\right) / n m \\
& <2\left(K+\Omega K+n m^{2} / 2 \Omega\right) / n m \\
& =2(\Omega+1) K / n m+m / \Omega .
\end{aligned}
$$

Here $\Omega$ is a free parameter such that $\Omega \geqslant 1$. Therefore we obtain the assertion of our theorem 1 . 


\section{Another estimate of $\lambda_{n+1}^{1}$}

Let $M$ be an $m(\geqslant 2)$-dimensional compact and oriented Riemannian manifold without boundary minimally immersed in the unit sphere (of any codimension). By means of (5), we have

$$
\langle\nabla \omega, \nabla \omega\rangle=\left\langle-\sum \nabla^{i} \nabla_{i} \omega, \omega\right\rangle \leqslant\langle\Delta \omega, \omega\rangle-\int_{M} \rho\left(\omega^{\sharp}, \omega^{\sharp}\right) d V_{M}
$$

for $\omega \in A^{1}(M)$. Here $\omega^{\sharp}$ denotes the vector field on $M$ such that $g\left(\omega^{\sharp}, X\right)=$ $\boldsymbol{\omega}(X)$ for any vector field $X$ on $M$. Thus we get

$$
\langle\nabla \omega, \nabla \omega\rangle \leqslant\langle\Delta \omega, \omega\rangle-r(M) \cdot\|\omega\|^{2} .
$$

Using (14) instead of (3), we have a similar argument as Section 2 to obtain the assertion of our theorem 2 .

Remark 3.1. Let $M$ be a Riemannian manifold as in theorem 1. Let $\left\{z^{j}\right\}$ be a normal coordinate system of $M$ about a point $z_{0} \in M$ as in proof of lemma 2.2. Then by means of (7), we have, at $z_{0}$,

$$
\rho\left(\omega^{\sharp}, \omega^{\sharp}\right)=\sum_{j}\left(m-1-\xi_{j}^{2}\right) \cdot \omega_{j}^{2} \quad\left(\omega \in A^{1}(M)\right) .
$$

Hence for any $\omega \in A^{1}(M)$, we have

$$
\left(m-1-S(M)^{2}\right) \cdot(\omega \mid \omega) \leqslant \rho\left(\omega^{\sharp}, \omega^{\sharp}\right) \leqslant(m-1) \cdot(\omega \mid \omega) .
$$

In particular, we get $m-1-S(M)^{2} \leqslant r(M)$. Thus for $p=1$, the inequality (1) follows from the inequality (2).

\section{Acknowledgment}

The author would like to express his sencere thanks to Professor $\mathrm{H}$. Kitahara and Professor T. Sakai who kindly have read through the manuscript to give advices.

\section{References}

[1] B. Y. Chen: Geometry of Submanifolds; Pure and Applied Mathematics 22, Marcel Dekker, Inc. New York (1973).

[2] S. Y. CHENG : Eigenfunctions and eigenvalues of laplacian; Proccedings of Symposia in Pure Mathematics, Vol. 27, 185-193 (1975).

[3] M. MAEdA: On the Eigenvalues of Laplacian; Science Reports of the Yokohama National University, No. 24, 29-33 (1977).

[4] L. E. PAyne, G. Pólya and H. F. Weinberger: On the ratio of consecutive eigenvalues; J. Math. and Phys. 35, 289-298 (1956). 
[5] G. de Rham: Variétés différentiable; Hermann Paris (1960).

[6] T. TAKahashi: Minimal immersions of Riemannian manifolds; J. Math. Soc. Japan, 18, 380-385 (1966).

Department of Mathematics

Faculty of Science

Hokkaido University

Sapporo, Japan. 